\title{
NOTE ON A FUNDAMENTAL LEMMA CONCERNING THE LIMIT OF A SUM*
}

\author{
BY H. J. ETTI.INGER
}

In a recent papert the author presented a proof of a lemma which is of fundamental importance in the theory of the Riemann integral. It is the purpose of this note to replace the proof of Case I of the above paper by the present one which treats the case of unequal subintervals. The theorem by means of which this proof is completed is of interest in itself, and admits of certain generalizations, one of which will be stated in the sequel.

A horizontal function of index $n, h(x, n)$, is a function defined on an interval $I \equiv(a \leqq x \leqq b)$ in such a manner that it is possible to divide $I$ into $n$ subintervals $I(i, n),(i=1,2, \cdots, n)$, so that $h(x, n) \equiv h_{i n} \equiv$ constant, at all interior points of $I(i, n)$. In addition, we define $h(-a, n) \equiv h_{1 n}$ and $h(b, n) \equiv h_{n n}$. Let $x(i, n)$ be the $n-1$ distinct internal points of division which subdivide $I$ and together with $a \equiv x(0, n), b \equiv x(n, n)$ form the subdivisions, $I(i, n)$, whose lengths are $\Delta_{i n}=x(i, n)$ $-x(i-1, n)$. We shall define the value of $h(x, n)$ at the internal points of division $x(i, n)$ to be that value of the pair of numbers $h_{i n}, h_{i+1, n}$, whose numerical value is the larger. Furthermore, let

$$
f(n)=\sum_{i=1}^{n} h_{i n} \Delta_{i n} .
$$

The fundamental lemma may now be restated as follows: Let $h(x, n)$ be a sequence of horizontal functions on $I$ for $n=1,2, \cdots$. Let $h(x, n)$ be bounded for all values of $n$ and for

* Presented to the Society, September 10, 1925.

+ An elementary proof of a fundamental lemma concerning the limit of a sum, this Bulletin, vol. 29 (1923), pp. 219-223. 
all values of $x$ in $I$, and let $\lim _{n \rightarrow \infty} h(x, n)=0$ for each fixed $x$ in I. Then $\lim _{n \rightarrow \infty} f(n)=0$.

We shall prove this lemma only for the special case (Case I of the earlier paper) that $|h(x, n+1)| \leqq|h(x, n)|$. From this case, the general case follows in accord with the proof given before. Let

$$
\bar{f}(n)=\sum_{i=1}^{n}\left|h_{i n}\right| \Delta_{i n}
$$

We have

$$
\bar{f}(n) \geqq|f(n)| \geqq 0,
$$

and

$$
\bar{f}(n+1) \leqq \bar{f}(n) .
$$

We shall show that given any $\epsilon>0$, there exists a positive integer, $N_{\epsilon}$, such that $|h(x, n)|<\epsilon$, for every $x$ in $I$ and $n \geqq N_{\text {e. }}$ Then $\bar{f}(n)<\epsilon(b-a)$ for $n \geqq N_{\text {e }}$. Hence $\bar{f}(n)$, and also $f(n)$, approach zero as a limit, as $n$ increases without limit.

Theorem. Given any $\epsilon>0$, there exists an $N_{6}$ such that $|h(x, n)|<\epsilon$ for every $x$ in $I$ and $n \geqq N_{\epsilon}$. Suppose the theorem false for the interval $I$. Let $L(i, n)$ be the subintervals into which $I$ is divided by adjacent pairs of points $x(i, j), i=$ $1,2, \cdots, j-1, j=1,2, \cdots, n$. If the theorem is false for the interval $I$, it must be false for at least one of the subintervals $L(1,2), L(2,2)$. Let $L\left(i_{2}, 2\right)$ be the first left hand one of these. If the theorem is false for $L\left(i_{2}, 2\right)$, it is false for at least one of the subintervals $L(i, 3)$ of $L\left(i_{2}, 2\right)$. Call the first left hand one of these $L\left(i_{3}, 3\right)$. Continuing this process, we obtain a monotonic non-increasing sequence of intervals, $L\left(i_{n}, n\right)$, which have therefore either a common interval $\bar{L}$ or at least a common point, $\bar{x}$. If $\bar{x}$ be regarded as representing either the common point, or one of the points of $\bar{L}$, all of which have a common $h(\bar{x}, n)$, then since $h(\bar{x}, n)$ approaches zero as a limit as $n$ increases indefinitely, $|h(\bar{x}, n)|<\epsilon$ for $n$ greater than a certain positive integer $N_{\bullet}$. This contradicts the hypo- 
thesis that the theorem is false for $I$, and establishes the desired result.

The above theorem may be stated otherwise as follows. If the sequence of horizontal functions $h(x, n)$ satisfying the conditions of the lemma approach the zero limit function monotonically, they must approach it uniformly. It follows as a corollary from this theorem that if we have a series of "interval" functions, such that $f(x, n)$ is a single-valued continuous curve in each subinterval, and if the sequence of functions approach a continuous limit function monotonically for each fixed $x$ in $I$, then the approach to the limit function is uniform with respect to $x$. In particular, the sequence of "interval" functions may be a set of functions $f_{n}(x)$ each of which is continuous in $I$.

UNIVERSITY OF TEXAS

\section{NOTE ON RATIONAL PLANE CUBICS*}

\section{BY C. A. NELSON}

1. Introduction. Many constructions have been devised for a rational plane cubic. One of the most interesting of them is due to Zahradnik ${ }^{+}$who noticed that the familiar construction for the cissoid of Diocles could be extended so as to generate any rational plane cubic. It is as follows : Take any conic $C$, a fixed point $O$ on $C$, and a fixed line $b$. Any line $l$ through $O$ meets $C$ a second time at $P$, and $b$ at $Q$. On $l$ lay off a segment $O M$ equal to and in the sense $P Q$. The locus of the point $M$ is a rational plane cubic $R$ with double point at $O$. The tangents to $R$ and $O$ are the joins of $O$ to the two points in which $b$ meets $C$.

Niewenglowskit showed a bit later that this same construction may be applied to $R$, using a second fixed line dis-

* Presented to the Society, September 10, 1925.

+ Zahradnik, Cissoidalcurven, Archiv der Mathematik und Physik, vol. 56, p. 8 .

† Niewenglowski, Sur les courbes d'ordre $n$ a point multiple d'ordre $n-1$, Comptes Rendus, vol. 80 (1875), p. 1067. 